\title{
Application of CFD in Studying and Modification of Gas Burners of Catalytic Reforming Unit of Abadan Refinery in Iran
}

\author{
Saeed Talebzadeh \\ Department of Gas \\ Engineering, Petroleum \\ university of Technology, \\ Ahvaz, Iran
}

\author{
Bahram Hashemi \\ Shahraki \\ Department of Gas \\ Engineering, Petroleum \\ university of Technology, \\ Ahvaz, Iran
}

\author{
Ahmad Shariati \\ Department of Gas \\ Engineering, Petroleum \\ university of Technology, \\ Ahvaz, Iran
}

\begin{abstract}
This article is a report of the attempts which were made to model an industrial premix gas burner which was operating in one of the furnaces of the Catalytic Reforming Unit (CRU) of Abadan refinery. The main objective of the modeling was to investigate on the effect of the structural geometry of the burner on its mode of operation, i.e. complete combustion of the gas, maximum generation of heat and production of a proper flame configuration. For this goal, fundamental equations of the conservation of mass, momentum and energy for fuel-air mixture flowing in the burner were written and solved by using the Computational Fluid Dynamic (CFD) techniques and the results were analyzed. The main conclusion of the research was that various effects of the changes in the burner come to appear and an appropriate correction on the burner was recommended to the industry.
\end{abstract}

\section{Keywords}

Computational Fluid Dynamics; Simulation; Gas Burners; Modification; Stoichiometric Ratio

\section{INTRODUCTION}

The development of advanced industrial burners with higher performance and higher efficiency is a major goal of combustion researchers, furnace designers and manufacturers. To reach the goal, on one hand, the new technical concepts and novelties for different processes have to be continuously developed. On the other hand, more efficient and economic tools, such as computer simulation by using computational fluid dynamics (CFD) technology, are also extremely important to be developed and applied for the design process and performance simulation of the new burners. ${ }^{1}$

To provide acceptable operation, the burner must be designed to perform following objects, commonly called $5-\mathrm{Ms}^{1,2}$ :

1. Meter the fuel and air into the flame zone.

2. Mix the fuel and air to efficiently utilize the fuel.

3. Maintain a continuous ignition zone for stable operation over the range.

4. Mold the flame to provide the proper flame shape.

5. Minimize pollutant emissions.

Mathematical modeling has been very widely used tools for the design of complicated burners since 80s. In 1988, a special issue of Combustion Science and Technology collected fourteen papers concerned with numerical methods for practical combusting flows and first formally illustrated their ability to contribute to the design process of industrial combustion equipment. Since then, both the digital computer technology and the numerical software have got very fast developed and are still growing quickly. Recently the computational fluid dynamic (CFD) tools, such as FLUENT and STAR-CD, etc., have been successfully used for comprehensive simulations of different types of burners, furnaces and boilers. ${ }^{1,3,4}$

CFD technology mainly consists of three parts. The first part is the physical models which are a set of conservation equations of mass, momentum, energy, state equation, turbulent equations, etc. The second part is a series of solution approaches for solving these physical models, and the third part is the preprocessor of discretization of computational domain and the postprocessor of visualization of numerical results. The early versions of commercial CFD codes usually applied the structured grid, which made the codes very difficult to deal with the industrial furnaces that may have very complicated geometry. Recently the new generation CFD codes, such as FLUENT 6, are based on the unstructured grid configurations, which make it possible to handle flexibly the very complicated geometry of an industrial burner. ${ }^{5}$

In this study CFD technology is used to simulate and evaluate the effects of some process variables and mechanical parameters on the efficiency of an industrial burner, such as impact of burner's tip draft variations on air to fuel ratio and on the fuel-air velocity at the burner's tip, effect of mass inflow variations on air to fuel ratio and tip velocity, effect of air controller opening on air to fuel ratio and tip velocity, effect of tip diameter on air to fuel ratio and tip velocity and finally effect of orifice diameter on air to fuel ratio and tip velocity. To do so, a new computational environment of Fluent 6 (which is one of the largest commercial CFD codes based on unstructured grid), was employed to solve the numerical equations for an industrial premix burner with a complicated geometry. The burner was a product of John Zink Company which is currently used in the Catalytic Reforming Unit of Abadan Refinery in Iran.

The technical approach in this work is comprehensive 3D numerical simulations for contours of pressure, contours of velocity, contours of species, air to fuel ratio, tip velocity and mixing of the fuel and air in the burner.

The composition of the fuel gas used in this burner is shown in Table 1. 
TABLE 1: Composition of the Natural Gas which was used in our analysis

\begin{tabular}{|c|c|}
\hline Components & Volume Percents \\
\hline $\mathrm{H}_{2} \mathrm{~S}$ & 0.70 \\
\hline $\mathrm{H}_{2}$ & 13.64 \\
\hline $\mathrm{C}_{1}$ & 61.91 \\
\hline $\mathrm{C}_{2}$ & 14.48 \\
\hline $\mathrm{C}_{3}$ & 6.41 \\
\hline $\mathrm{i}-\mathrm{C}_{4}$ & 0.94 \\
\hline $\mathrm{n}-\mathrm{C}_{4}$ & 1.06 \\
\hline $\mathrm{i}-\mathrm{C}_{5}$ & 0.52 \\
\hline $\mathrm{n}-\mathrm{C}_{5}$ & 0.27 \\
\hline $\mathrm{C}_{6}{ }^{+}$ & 0.07 \\
\hline
\end{tabular}

$\mathrm{C}_{6}^{+}$is the sum of mole fraction of hexane and heavier hydrocarbons.

GAMBIT software was used to produce the geometry of the burner. At first, dimensions of the burner are fed to GAMBIT software as inputs. Figure 1 shows the dimensions of the burner portions. Result is a three dimensional geometry of the burner. This geometry is then imported to CFD software (i.e. FLUENT). Since the CFD software solves the governing equation numerically, griding of the burner is also made by GAMBIT software. The tetrahedron grids were used for gridding the geometry of the burner.

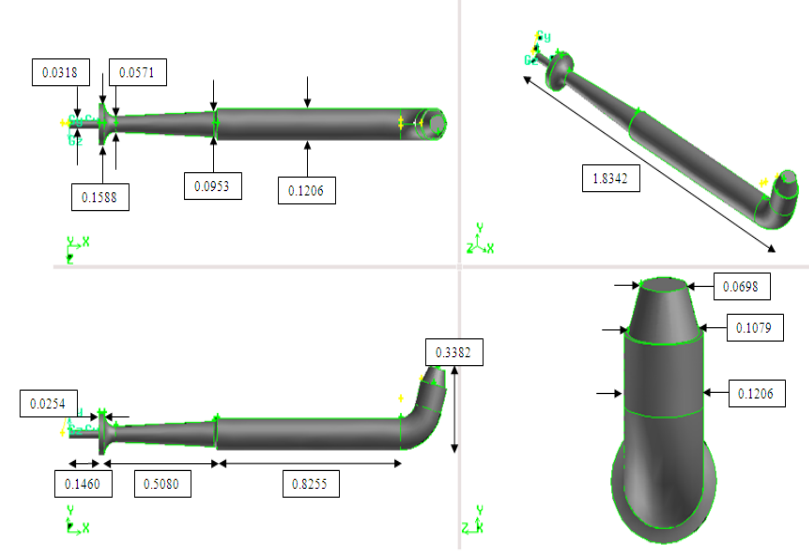

FIGURE 1: Size of the Burner in Meter (m)

\section{THEORY}

In this study an industrial premix gas burner which was operating in one of the furnaces of the Catalytic Reforming Unit (CRU) of Abadan refinery was modeled. FLUENT software was used for solving numerical equations using CFD technique. As the following sections will indicate, the equations are complex because they have to be simultaneously dealt with several interrelated transport mechanisms. Needless to say, they are also difficult to solve for complex geometries. The equation for conservation of mass, or continuity equation, can be written as follows:

$\frac{\partial \rho}{\partial \mathrm{t}}+\nabla \cdot(\rho \vec{v})=\mathrm{S}_{\mathrm{m}}$

Equation 1 is the general form of the mass conservation equation and is valid for incompressible as well as compressible flows. The source $S_{m}$ is the mass added to the continuous phase from the dispersed second phase (e.g., due to vaporization of liquid droplets) and any user-defined sources. ${ }^{6,7}$ Since our model works in steady state and there is no additional source, hence the equation 1 simplifies to equation 2:

$\nabla .(\rho \vec{v})=\mathbf{0}$

Conservation of momentum in an inertial (non-accelerating) reference frame is described by

$$
\frac{\partial}{\partial t}(\rho \vec{v})+\nabla \cdot(\rho \vec{v} \vec{v})=-\nabla p+\nabla \cdot(\overline{\bar{\tau}})+\rho \vec{g}+\vec{F}
$$

Where $\boldsymbol{p}$ is the static pressure, $\overline{\overline{\boldsymbol{\tau}}}$ is the stress tensor (described below), and $\boldsymbol{\rho} \overrightarrow{\boldsymbol{g}}$ and $\overrightarrow{\boldsymbol{F}}$ are the gravitational body force and external body forces (e.g., that arise from interaction with the dispersed phase), respectively. $\overrightarrow{\boldsymbol{F}}$ also contains other modeldependent source terms such as porous-media and userdefined sources. The stress tensor $\overline{\overline{\boldsymbol{\tau}}}$ is given by

$\overline{\bar{\tau}}=\mu\left(\nabla \vec{v}+\nabla \vec{v}^{T}-\frac{2}{3} \nabla \cdot \vec{v} I\right)$

Where $\boldsymbol{\mu}$ is the molecular viscosity, $\boldsymbol{I}$ is the unit tensor, and the second term on the right hand side is the effect of volume dilation. ${ }^{8,9}$ In this study; our system is at steady state and there is no external force and volume dilation; because velocity of the gas is nearly high, the effect of the gravity is neglected. Hence equations 3 and 4 simplify to the fallowing equation:

$\nabla \cdot(\rho \vec{v} \vec{v})=-\nabla p+\nabla \cdot\left[\mu\left(\nabla \vec{v}-\frac{2}{3} \nabla \cdot \vec{v} I\right)\right]$

The turbulence kinetic energy, $k$, and its rate of dissipation, $\varepsilon$, are obtained from the following transport equations:

$\frac{\partial}{\partial t}(\rho k)+\frac{\partial}{\partial x_{i}}\left(\rho k u_{i}\right)=\frac{\partial}{\partial x_{i}}\left[\left(\mu+\frac{\mu_{t}}{\sigma_{k}}\right) \frac{\partial k}{\partial x_{i}}\right]+G_{k}+G_{b}-$ $\rho \epsilon-Y_{m}+S_{k}$

$\frac{\partial}{\partial t}(\rho \epsilon)+\frac{\partial}{\partial x_{i}}\left(\rho \epsilon u_{i}\right)=\frac{\partial}{\partial x_{i}}\left[\left(\mu+\frac{\mu_{t}}{\sigma_{\epsilon}}\right) \frac{\partial \epsilon}{\partial x_{i}}\right]+$

$C_{1 \epsilon} \frac{\epsilon}{k}\left(G_{k}+C_{3 \epsilon} G_{b}\right)-C_{2 \epsilon} \rho \frac{\epsilon^{2}}{k}+S_{\epsilon}$

In these equations, $\boldsymbol{G}_{\boldsymbol{k}}$ represents the generation of turbulence kinetic energy due to the mean velocity gradients. $\boldsymbol{G}_{\boldsymbol{b}}$ is the generation of turbulence kinetic energy due to buoyancy. $\boldsymbol{Y}_{\boldsymbol{M}}$ represents the contribution of the fluctuating dilatation in compressible turbulence to the overall dissipation rate. $\boldsymbol{C}_{\boldsymbol{l}}$, $C_{2 \varepsilon}$, and $C_{3 \varepsilon}$ are constants. $\sigma_{k}$ and $\sigma_{\varepsilon}$ are the turbulent Prandtl numbers for $\boldsymbol{k}$ and $\boldsymbol{\epsilon}$, respectively. $\boldsymbol{S}_{\boldsymbol{k}}$ and $\boldsymbol{S}_{\varepsilon}$ are user-defined source terms. ${ }^{10}$ In the steady state systems the first terms of these equations is eliminated. Since in our system buoyancy and compressibility neglected, $\boldsymbol{G}_{\boldsymbol{b}}$ and $\boldsymbol{Y}_{\boldsymbol{M}}$ are equal to zero. Thus, equations 6 and 7 simplify to equations 8 and 9 , respectively.

$\frac{\partial}{\partial x_{i}}\left(\rho k u_{i}\right)=\frac{\partial}{\partial x_{i}}\left[\left(\mu+\frac{\mu_{t}}{\sigma_{k}}\right) \frac{\partial k}{\partial x_{i}}\right]+G_{k}-\rho \epsilon$

$\frac{\partial}{\partial x_{i}}\left(\rho \epsilon u_{i}\right)=\frac{\partial}{\partial x_{i}}\left[\left(\mu+\frac{\mu_{t}}{\sigma_{\epsilon}}\right) \frac{\partial \epsilon}{\partial x_{i}}\right]+C_{1 \epsilon} \frac{\epsilon}{k} G_{k}-C_{2 \epsilon} \rho \frac{\epsilon^{2}}{k}$

The turbulent (or eddy) viscosity, $\boldsymbol{\mu}_{\boldsymbol{t}}$, is computed by combining $k$ and $\varepsilon$ as follows:

$\mu_{t}=\rho C_{\mu} \frac{k^{2}}{\epsilon}$

The model constants $\boldsymbol{C}_{\mathbf{1} \boldsymbol{\epsilon}}, \boldsymbol{C}_{\mathbf{2} \boldsymbol{\epsilon}}, \boldsymbol{C}_{\boldsymbol{\mu}}, \boldsymbol{\sigma}_{\boldsymbol{k}}, \boldsymbol{\sigma}_{\boldsymbol{\epsilon}}$ and $\boldsymbol{C}_{\boldsymbol{\mu}}$ have the following default values: $C_{1 \epsilon}=1.44, C_{2 \epsilon}=1.92, C_{\mu}=$ $0.09, \sigma_{k}=1.0, \sigma_{\epsilon}=1.3$

The energy equation is in the following form: $\frac{\partial}{\partial t}(\rho E)+\nabla \cdot(\vec{v}(\rho E+p))=\nabla \cdot\left(k_{e f f} \nabla T-\sum_{j} h_{j} \vec{J}_{J}+\right.$ $\left.\left(\overline{\overline{\boldsymbol{\tau}}}_{\text {eff }} \cdot \overrightarrow{\boldsymbol{v}}\right)\right)+\boldsymbol{S}_{\boldsymbol{h}}$

Where $\mathrm{k}_{\mathrm{eff}}$ is the effective conductivity $\left(k+k_{\mathrm{t}}\right.$, where $k_{t}$ is the turbulent thermal conductivity, defined according to the 
turbulence model being used), and $\overrightarrow{J_{j}}$ is the diffusion flux of species $j$. The first three terms on the right-hand side of Equation 11 represent energy transfer due to conduction, species diffusion, and viscous dissipation, respectively. $S_{h}$ includes the heat of chemical reaction, and any other volumetric heat sources we have defined. In Equation 11

$E=h-\frac{p}{\rho}+\frac{v^{2}}{2}$

Where sensible enthalpy $h$ is defined for ideal gases as

$\boldsymbol{h}=\sum_{j} \boldsymbol{Y}_{\boldsymbol{j}} \boldsymbol{h}_{\boldsymbol{j}}$

And for incompressible flows as

$\boldsymbol{h}=\sum_{j} \boldsymbol{Y}_{\boldsymbol{j}} \boldsymbol{h}_{j}+\frac{\boldsymbol{p}}{\rho}$

In Equations 13 and $14, Y_{j}$ is the mass fraction of species $j$ and

$h_{j}=\int_{T_{r e f}}^{T} c_{p, j} d T$

Where $\mathrm{T}_{\text {ref }}$ is $298.15 \mathrm{~K}^{6}$

In our study, the model is steady state and since the viscosity of gases is low, viscous heating is negligible and, there is no chemical reaction either. Hence equation 11 simplifies to the fallowing equation:

$\boldsymbol{\nabla} \cdot(\overrightarrow{\boldsymbol{v}}(\boldsymbol{\rho} E+\boldsymbol{p}))=\boldsymbol{\nabla} \cdot\left(\boldsymbol{k}_{\text {eff }} \boldsymbol{\nabla T}-\sum_{j} \boldsymbol{h}_{j} \overrightarrow{J_{j}}\right)$

When conservation equations are solved for chemical species, FLUENT predicts the local mass fraction of each species, $Y_{i}$, through the solution of a convection-diffusion equation for the $i$ th species. This conservation equation takes the following general form: ${ }^{6,7,11}$

$\frac{\partial}{\partial t}\left(\rho Y_{i}\right)+\nabla \cdot\left(\rho \vec{v} Y_{i}\right)=-\nabla \cdot \overrightarrow{J_{l}}+R_{i}+S_{i}$

Where $R_{i}$ is the net rate of production by chemical reaction and $S_{i}$ is the rate of creation by addition from the dispersed phase plus any user-defined sources. ${ }^{3}$ For the steady state system, the first term on the right hand side of the equation eliminates and since there is no additional source in the model and no reaction take place in a premix burner, so species transport equation simplifies to the following equation:

$\nabla \cdot\left(\rho \vec{v} Y_{i}\right)=-\nabla \cdot \overrightarrow{J_{1}}$

In turbulent flows, FLUENT computes the mass diffusion in the following form:

$\overrightarrow{J_{\imath}}=-\left(\rho D_{i, m}+\frac{\mu_{t}}{S c_{t}}\right) \nabla Y_{i}$

Where $\mathrm{Sc}_{\mathrm{t}}$ is the turbulent Schmidt number.

For many multicomponent mixing flows, the transport of enthalpy due to species diffusion

$$
\nabla .\left[\sum_{i=1}^{n} h_{i} \overrightarrow{J_{l}}\right]
$$

can have a significant effect on the enthalpy field and should not be neglected. In particular, when the Lewis number

$\boldsymbol{L} \boldsymbol{e}_{\boldsymbol{i}}=\frac{\boldsymbol{k}}{\rho c_{p} D_{i, m}}$

for any species is far from unity, neglecting this term can lead to significant errors. FLUENT will include this term by default. In Equation 20, $k$ is the thermal conductivity. ${ }^{6}$

\section{RESULTS AND DISCUSSIONS}

For all of fluid dynamics problems, the continuity and momentum equations must be solved. Since there are several components in the fuel gas, for mixing effect, the energy equation is also required. For high velocity gas in orifice (input of the burner), the turbulence flow is modeled using k- $\varepsilon$ method. The sound velocity is close to $400 \mathrm{~m} \mathrm{~s}-1$ and the velocity of the gas fuel is less than $40 \mathrm{~m} \mathrm{~s}-1$, so the Mach number is less than 0.1 . In this condition, compressibility effect is not considered in burner modeling. For each component the material balance should be solved and mixing laws are used for calculating the physical properties of the gas mixtures. Ambient temperature and fuel gas temperature are 313 Kelvin and 305 Kelvin, respectively. Rate of gas inflow is equal to $0.00375 \mathrm{~kg} \mathrm{~s}-1$.

FLUENT was used as simulating software. Solution of differential equations in this software is based on the control volume methods.

In this software, the geometry of the burner is imported from Gambit software as an input and it is checked. Then solver is selected and governing equations, which were discussed in detail, are defined for the burner. Next, the boundary conditions and gas properties must be carefully defined. After completing the definition of the model, the solution parameters are set and then FLUENT solves the problem.

Figure 2 shows the contours of mass fraction of each species. For $\mathrm{H}_{2} \mathrm{~S}, \mathrm{H}_{2}, \mathrm{CH}_{4}, \mathrm{C}_{2} \mathrm{H}_{6}, \mathrm{C}_{3} \mathrm{H}_{8}, \mathrm{C}_{4} \mathrm{H}_{10}$ and $\mathrm{C}_{5} \mathrm{H}_{12}$, mass fraction can be found from upper shape of the Figure 2 and for $\mathrm{O}_{2}$ and $\mathrm{N}_{2}$ from the lower shape of the Figure 2. For example to find the mass fraction of $\mathrm{O}_{2}$ in inlet air, one can look to the lower shape and find the red color. Red color in the table of Figure 2 shows a mass fraction for $\mathrm{O}_{2}$ is 0.23 that it is the mass fraction of $\mathrm{O}_{2}$ in the air. According to Figure 2, at the end of the nozzle, the mass fractions of all the species are fixed. It is concluded that the complete mixing of the air and fuel are achieved.

\subsection{Impact of Tip Burner Draft Variations on Air to Fuel Ratio and Tip Velocity}

In this study, the actual furnace draft value was not implemented in primary simulation calculations because of lack of data. However it is known that natural draft normally is less than 200 Pascal. So, the effect of draft was investigated on air to fuel ratio and tip velocity. The natural draft variation range considered to be from 33 to 200 Pascal. The results are shown in Figure 3.
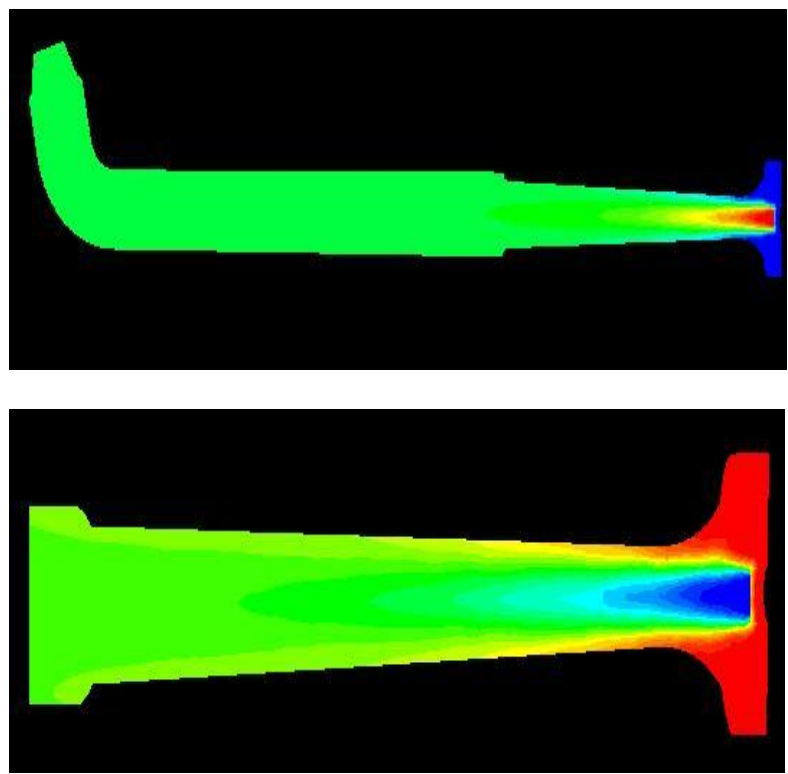

\begin{tabular}{|l|l|l|l|l|} 
Color & $\mathbf{H}_{2} \mathrm{~S}$ & $\mathbf{H}_{2}$ & $\mathbf{C H}_{4}$ & $\mathbf{C}_{2} \mathbf{H}_{6}$ \\
\hline
\end{tabular}




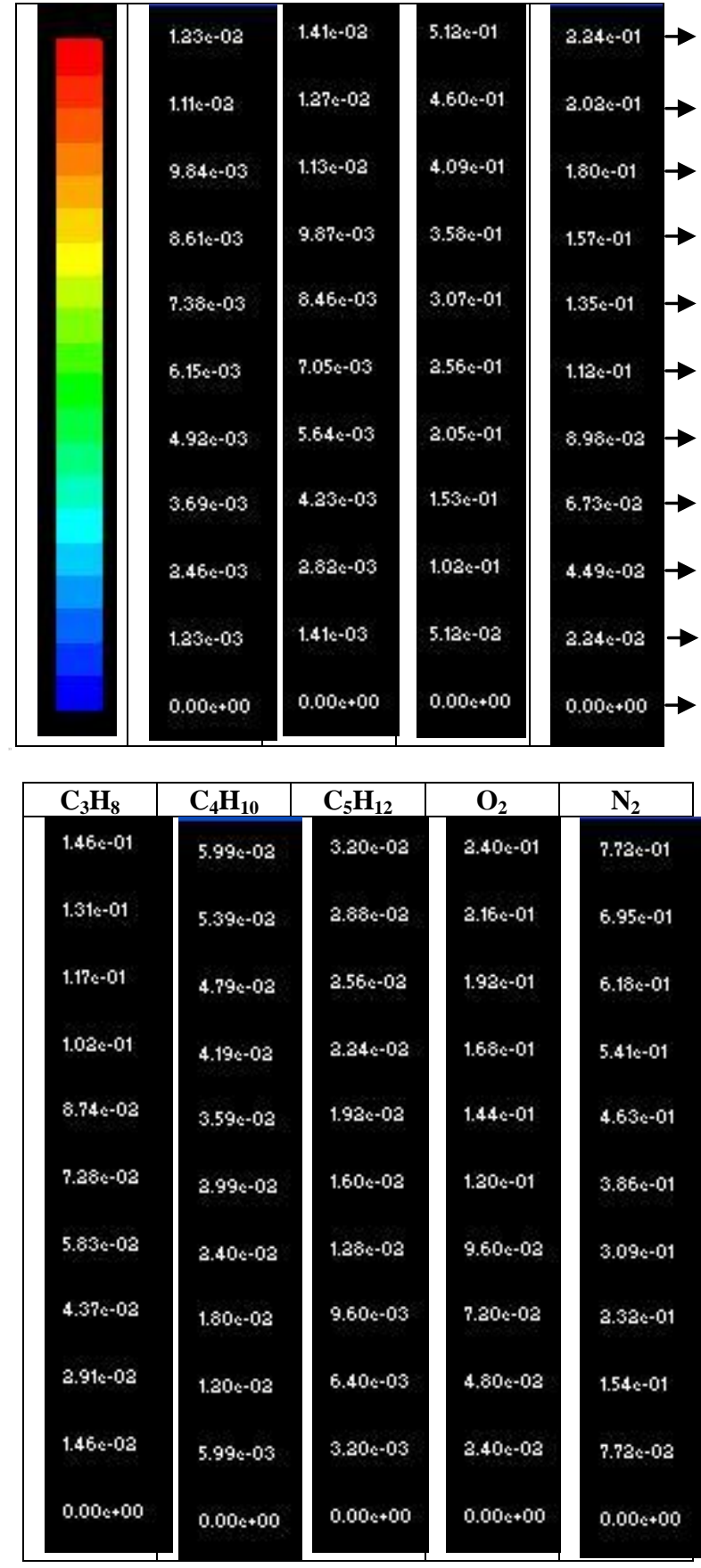

FIGURE 2: Contours of mass fraction species; For $\mathrm{H}_{2} \mathrm{~S}, \mathrm{H}_{2}, \mathrm{CH}_{4}, \mathrm{C}_{2} \mathrm{H}_{6}, \mathrm{C}_{3} \mathrm{H}_{8}, \mathrm{C}_{4} \mathrm{H}_{10}$ and $\mathrm{C}_{5} \mathrm{H}_{12}$, mass fraction can be found from the upper figure and for $\mathrm{O}_{2}$ and $\mathrm{N}_{2}$ mass fraction can be found from the lower figure.

Increased draft at the tip of the burner sucks more air to the burner. Since the gas flow rate is constant, the increased rate of the air increases the air to fuel ratio and exhausted gas velocity at the tip. The simulation results show that there is an approximate linear relationship between air to fuel ratio and the furnace draft. The same is true for the exhausted gas velocity at the burner tip and the furnace draft.
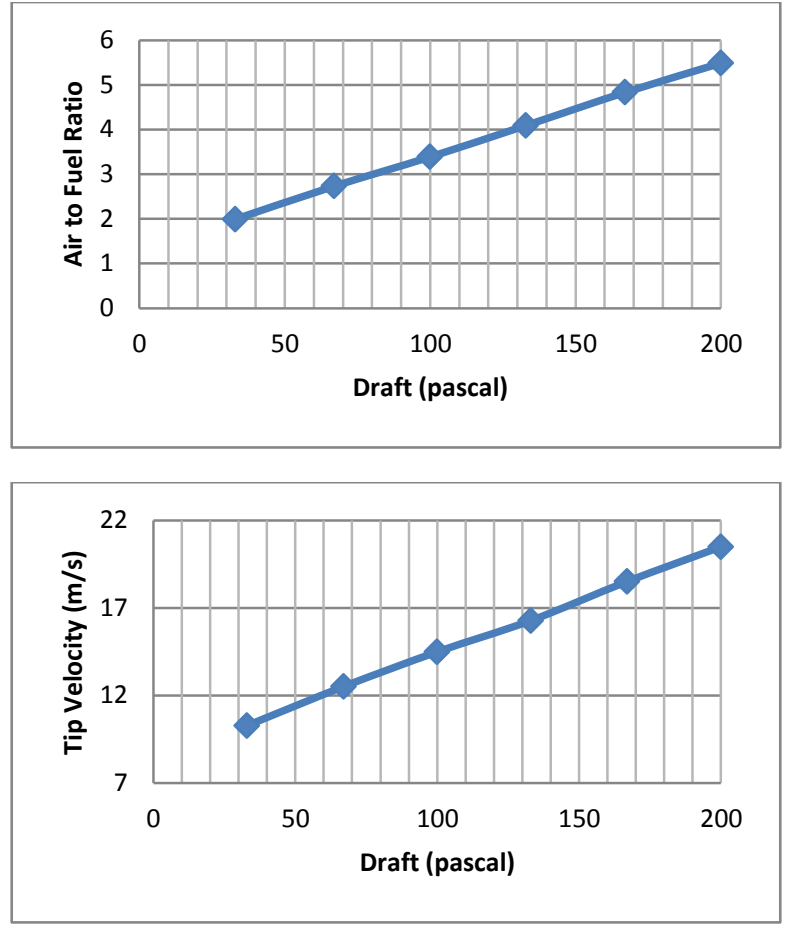

FIGURE 3: Impact of tip burner draft variation on air to fuel ratio and tip velocity

\subsection{Effect of Mass Inflow Variations on Air to Fuel Ratio and Tip Velocity}

The gas mass flow rate to the burner is less than its designed value. It might be a reason for the furnace operating at low efficiency. The gas flow rate was increased to its design values in the simulations. The effect of this increase on air to fuel ratio and tip velocity are shown in Figure 4.
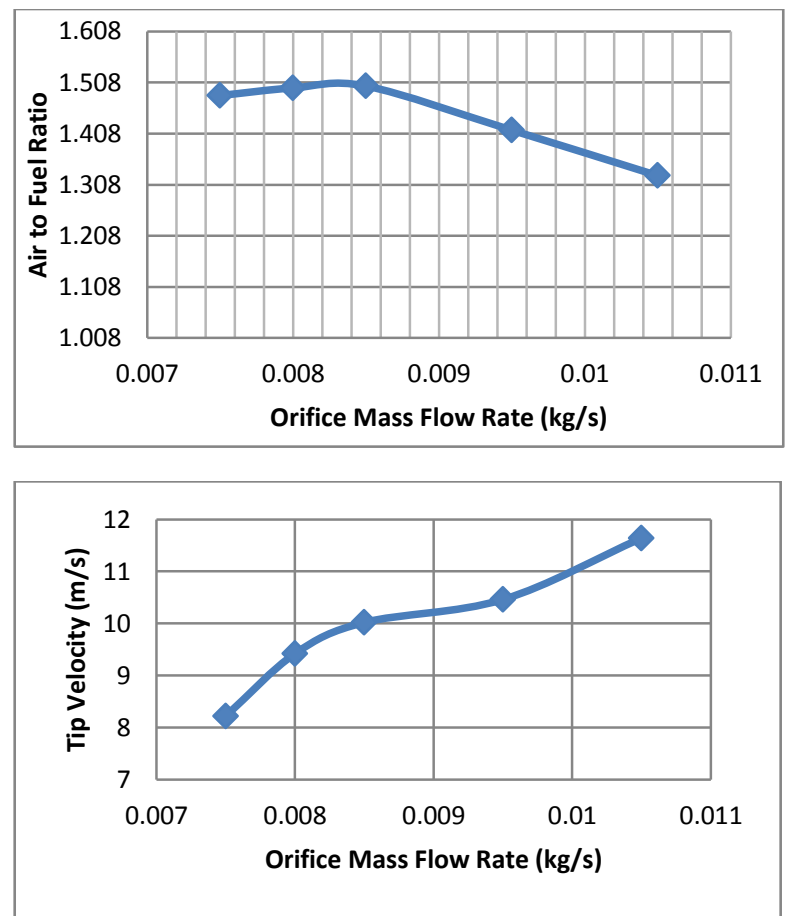

FIGURE 4: Effect of mass inflow variations on air to fuel ratio and tip velocity 
In Figure 4 air to fuel ratio increases until it reaches to a maximum and then levels off. The increase in air to fuel ratio is the result of draft increasing in venturi throat. The decrease in air to fuel ratio is justified by increase of exit gas from the orifice which prevent the air entering to the burner. However, increasing the mass inflow does not change the air to fuel ratio significantly. Increasing the mass inflow, increase the tip velocity as it is expected.

\subsection{Effect of Air Controller Opening on Air to Fuel Ratio and Tip Velocity}

According to the feed gas composition, air to fuel ratio equal to 18.37 is required for complete combustion. On the other hand the primary inlet air usually must be $50-60 \%$ of the required air. So, the air to fuel ratio must be in the range 9.185 to 11.022. Simulation result with 200 Pascal draft gives a 5.5 air to fuel ratio which is less than the amount for the complete combustion. The effect of air controller on air to fuel ratio was investigated. The results of such simulations are shown in Figure 5.

According to the results shown in Figure 5, it is seen that amount of opening of the air regulation plate has very little effect on the air to fuel ratio and on the exhausted gas from the burner tip.
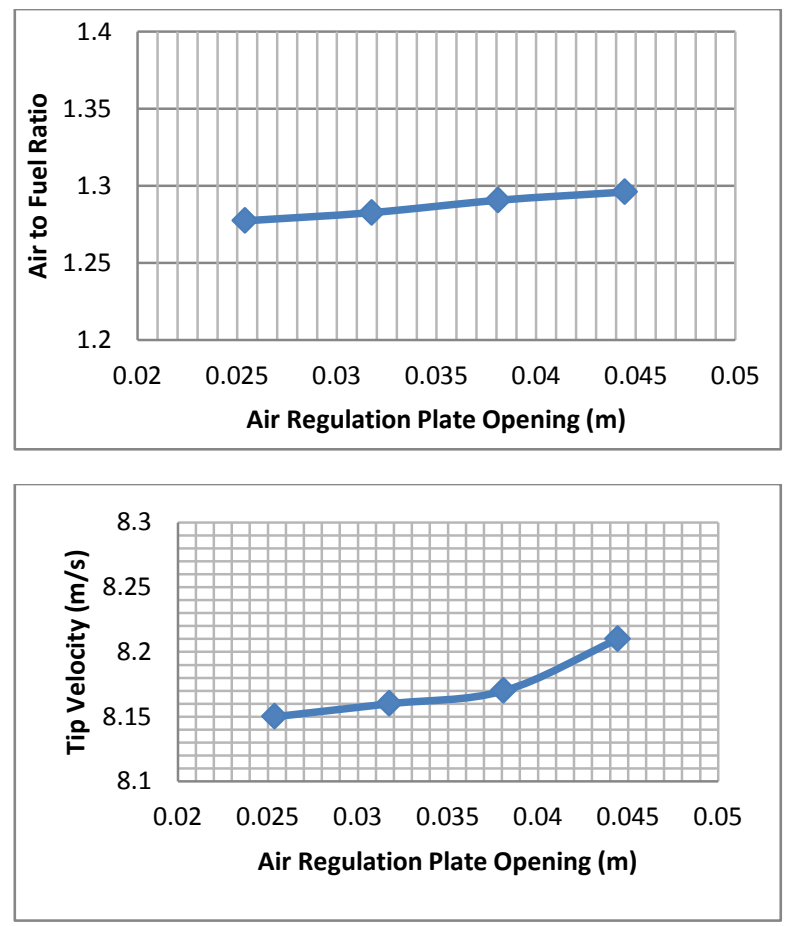

FIGURE 5: Effect of air controller opening on air to fuel ratio and tip velocity

\subsection{Effect of Tip Diameter on Air to Fuel Ratio and Tip Velocity}

The effect of variation of tip diameter on air to fuel ratio and tip velocity are shown in Figure 6. As it is expected, decrease in tip diameter will increase the tip velocity. It also increases the pressure in the burner which in turn reduces the amount of the sucked air to the burner. So for increasing the amount of the air to fuel ratio the tip burner diameter should be increased.
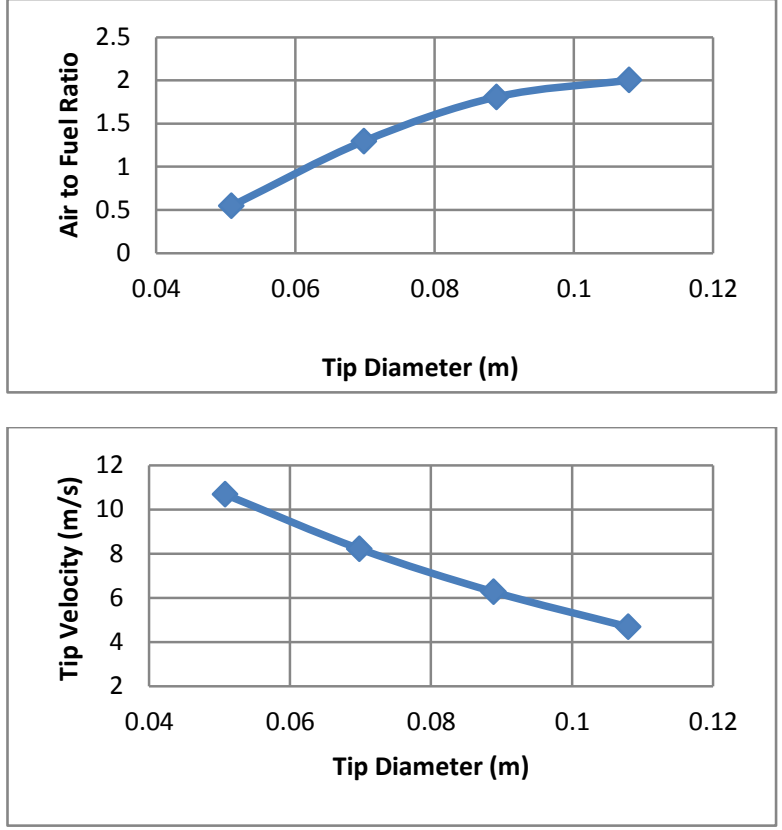

FIGURE 6: Effect of tip diameter on air to fuel ratio and tip velocity

\subsection{Effect of Orifice Diameter on Air to Fuel Ratio and Tip Velocity}

As the orifice diameter decreases, the gas velocity increases and the pressure decreases. The decrease in pressure increases the amount of sucked air to the burner that results to an increase in air to fuel ratio.
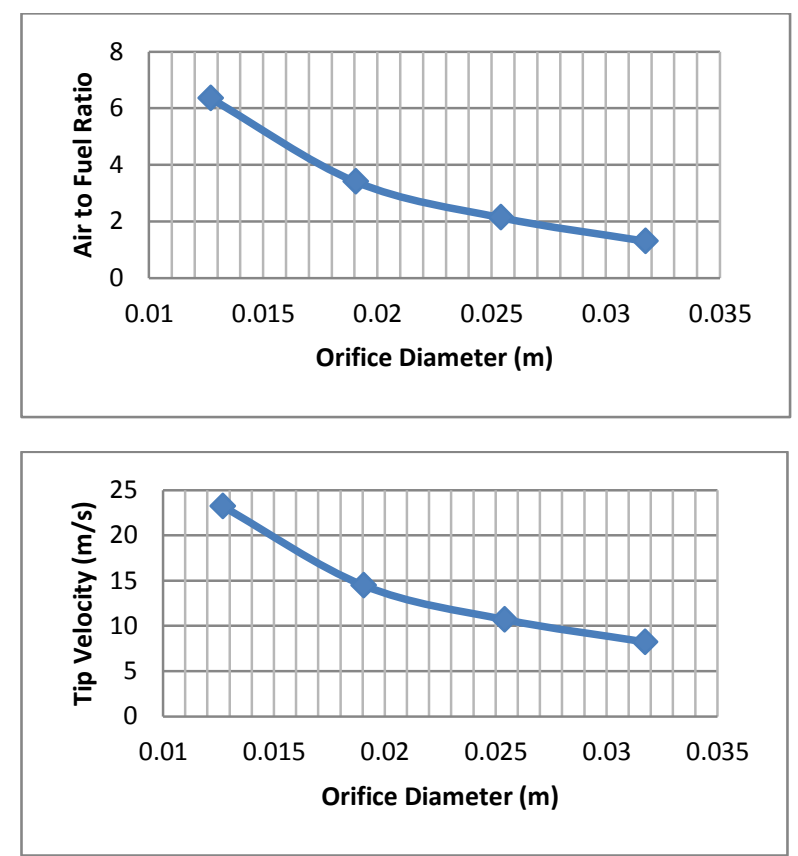

FIGURE 7: Effect of orifice diameter on air to fuel ratio

As can be seen in Figure 7 the effect of orifice diameter on air to fuel ratio and tip velocity is significant. So this parameter can be used for controlling the air which is needed for complete burning of the gas fuel. 


\subsection{Combining the Effects}

The simulation results show that the amount of entrained air to the burner is low. On the other hand it shows that the effect of the tip burner draft variation, the effect of the tip diameter and the effect of the orifice diameter on air to fuel ratio and on tip velocity are great. So, by combining these effects we can supply the amount of oxygen that is required for complete burning of the fuel gas, and thereby save in the fuel and energy consumption rate. The results of combined effects are shown in Table 2.

TABLE 2: Air to Fuel Ratio and Tip Velocity for Combined Changes in Burner Parameters

\begin{tabular}{|c|c|c|c|c|c|}
\hline Parameters & Existing Condition & Comb.1 & Comb.2 & Comb.3 & Comb. 4 \\
\hline Gas mass flow rate (kg s-1) & 0.0075 & 0.0105 & 0.0075 & 0.0075 & 0.0075 \\
\hline Draft at the tip of burner (assumed) (Pascal) & 33 & 100 & 100 & 100 & 100 \\
\hline Tip diameter (m) & 0.0698 & 0.0698 & 0.0698 & 0.1079 & 0.0698 \\
\hline Orifice diameter (m) & 0.0318 & 0.0318 & 0.0318 & 0.0318 & 0.0190 \\
\hline Air regulator opening length (m) & 0.0254 & 0.0254 & 0.0444 & 0.0254 & 0.0254 \\
\hline Air to fuel ratio (mass ratio) & 1.99 & 2.44 & 3.34 & 5.04 & 4.78 \\
\hline Velocity of the gas at the tip of the burner (m s-1) & 8.22 & 16.30 & 14.33 & 4.78 & 18.64 \\
\hline
\end{tabular}

According to the theoretical calculations, the air to fuel ratio must be in the range 9.185 to 11.022 . Hence for this approach for an example, some parameters are varied and a new model is defined as follows:

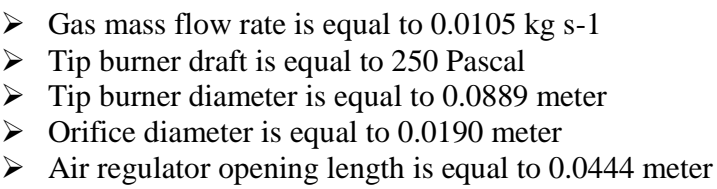

With these variations of parameters, result of the simulation shows that the air to fuel ratio is equal to 10.41 , that is at acceptable range.

\section{CONCLUSIONS}

This study led us to the following conclusions:

1. Development of the contours of the mass fraction of the components fuel gas/air mixture in the burner, contours of static pressure, contours of static temperature and contours of the absolute velocity of the mixture in the venturi part of the burner by using CFD technique to a better insight through the process that was going on in the burner. It was found that the induced draft at the venturi throat was not enough to suck sufficient primary air into the burner and consequently the entrained air by the gas was low.

2. Simulation of the model revealed that it is possible to increase the air to gas mass flow ratio and the tip velocity if the furnace draft could be increased and monitored properly. It was also led to:

- Tip velocity increases by increasing gas to the burner but air to fuel ratio is subject to some limitations. It increases first due to the increased draft, while then it decreases smoothly when the gas flow rate is increased from some specified value.

- At the current condition the air opening plate displacement cannot alter the air to fuel ratio and the tip velocity considerably.

- Tip velocity decreases with increasing tip diameter while air to fuel ratio increases well.

- Air to fuel ratio increases with decreasing the gas orifice diameter. The increases of the air mass flow rate due to the increased induced draft compensate the decrease of the gas flow rate so that an increase in the tip velocity is the ultimate result when the gas orifice diameter is decreased.

3. Simulation results show that it was possible to modify the existing burners so that a proper air to fuel ratio and a suitable burning is attained. The burners tip diameter, the gas orifice diameter and the draft in the furnace alternations are needed to be done for this goal.

\section{ACKNOWLEDGMENTS}

I would like to thank Abadan Refinery (R\&D office) for their informational and financial support of this project.

\section{REFERENCES}

[1] Charles E. Baukal, JR., PH.D. ,P.E., John Zink Company, LLC, Tulsa, Oklahama (2001), The John Zink Combustion Handbook, Boca Raton London New York Washington, D.C., CRC Press.

[2] A. Garg, Better burner specifications, Hydrocarbon Processing, 68(8), 71-72, 1989.

[3] API Publication 535: Burner for Fired Heaters in General Refinery Services, 1st edition, American Petroleum Institute, Washington, D.C., July 1995.

[4] S.R. Turns, An Introduction to Combustion, McGraw-Hill, New York, 1996.

[5] Wei dong, KTH Royal Institute of Technology, Design of Advanced Industrial Furnaces Using Numerical Modeling Method, Stockholm, March 2000.

[6] FLUENT v.6 Help, Fluent Inc. 2001-11-29.

[7] G. K. Batchelor. An Introduction to Fluid Dynamics. Cambridge Univ. Press, Cambridge, England, 1967.

[8] G. K. Batchelor. An Introduction to Fluid Dynamics. Cambridge Univ. Press, Cambridge, England, 1967.

[9] F. Kreith, Ed., The CRC Handbook of Mechanical Engineering, CRC Press, Boca Raton, FL, 1998.

[10] B. E. Launder and D. B. Spalding. Lectures in Mathematical Models of Turbulence. Academic Press, London, England, 1972.

[11] W. C. Reynolds. Fundamentals of turbulence for turbulence modeling and simulation, Lecture Notes for Von Karman Institute Agard Report No. 755, 1987. 